\title{
МОДУЛЯЦИЯ ЭЛЕКТРОННОЙ СТРУКТУРЫ УЛЬТРАКОРОТКОЙ УГЛЕРОДНОЙ НАНОТРУБКИ (0,9) ПРИ ЛЕГИРОВАНИИ ЩЕЛОЧНЫМИ МЕТАЛЛАМИ
}

\author{
() 2017 Г. И. Глушков, А. В. Тучин, Е. Н. Бормонтов \\ Воронежский государственный университет, Университетская пл., 1, 394018 Воронеж, Россия \\ e-mail: green5708@yandex.ru
}

Поступила в редакцию 14.08.2017

\begin{abstract}
Аннотация: В работе установлено, что в узком интервале длин в металлических трубках $(0,9)$ не выполняется правило $3 k$, существует ненулевая запрещенная зона, и трубки становятся полупроводниковыми. Энергетический зазор между граничными орбиталями в триплетном состоянии зависит от спина, поэтому проводимость трубки спинзависима, что обуславливает перспективу использования ук-УНТ в устройствах со спиновым транспортом. Ширина запрещенной зоны убывает при дискретном наращивании длин трубки.

Контролируемое введение металлической примеси приводит к модуляции запрещенной зоны, росту проводимости и спиновой поляризации в случае анионных комплексов. Таким образом, показан технологический способ управления свойствами материала для создания функциональных устройств спинтроники.
\end{abstract}

Ключевые слова: спин, спинтроника, углеродная нанотрубка, допирование, щелочные металлы.

\section{ВВЕДЕНИЕ}

Динамичное развитие электроники, индустрии сверхбыстродействующих систем привело к выделению новых отраслей, среди которых и спиновая электроника (спинтроника), использующая не только зарядовые, но и спиновые свойства носителей. Основной задачей спинтроники является поиск материалов, в которых возможно достичь высокой степени спиновой поляризации, протекания спинполяризованных токов без инверсии поляризации и механизмов управления поляризацией для конструирования функциональных устройств.

В качестве таких материалов активно исследуется широкий спектр наноструктур и композитов, включая углеродные структуры. Существенные успехи достигнуты в создании функциональных спинтронных устройств на основе графеновых нанолент сложной геометрии, однако изготовление их крайне технологически сложно, а получаемые структуры нестабильны. Управление степенью поляризации осуществлялось сильными магнитными и электрическими полями [1-10].

Рассматривая возможности применения ультракоротких углеродных нанотрубок (ук-УНТ) с металлической проводимостью в качестве функ- ционального материала спинтроники, следует отметить следующие преимущества: в достаточно узком интервале длин квантово-химические ограничения приводят к наличию ненулевого энергетического зазора между граничными орбиталями, т. е. классическое правило $3 k$ не выполняется. В последние годы разработаны методики синтеза ук-УНТ с узким распределением по длине и хиральности трубок, структура которых термодинамически стабильна.

В ряде работ по исследованию электронной структур ук-УНТ показано, что в узком интервале длин достигается значительная спиновая поляризация, показана возможность управления ее величиной приложением внешнего стационарного электрического поля. Другим технологическим инструментом для модуляции спиновой поляризации является контролируемое введение примесей. Современные методики синтеза предполагают использование металлических катализаторов, т. е. в процессе роста производится контролируемое введение металлических примесей, именно поэтому целью данной работы является теоретическое исследование модуляции параметров электронной структуры при допировании углеродных нанотрубок металлическими примесями. 


\section{ДЕТАЛИ РАСЧЕТОВ}

Закрытая зигзагообразная ук-УНТ $(0,9)$ состоит из двух шапок $\mathrm{C}_{30}$ и цилиндрического остова с переменным числом кольцевых сегментов, содержащих по 18 атомов углерода. При нулевом числе сегментов ук-УНТ $(0,9)$ представляет собой фуллерен $\mathrm{C}_{60}$, хорошо изученный теоретически и экспериментально. Стехиометрическая формула закрытой ук-УНТ $(0,9) \mathrm{C}_{60+18 i}$. Нанотрубка имеет симметрию $D_{3 \mathrm{~d}}$ при $i=2 k, D_{3 h}$ симметрию при $i=2 k+1$ и $D_{3}$ при любых $i(k=0,1,2, \ldots)$ (рис. 1). Изменение длины трубки при расчетах производится дискретным увеличением числа сегментов.

Отсутствие трансляционной симметрии в укУНТ определяет использование молекулярного приближения при исследовании их электронной структуры. Наибольшее распространение для моделирования ук-УНТ получила теория функционала электронной плотности (DFT) [9-21]. В настоящей работе исследование электронной структуры ук-УНТ в основном состоянии проводилось методом DFT с использованием обменно-корреляционных функционалов LSDA и B3LYP в базисах $3-21 * \mathrm{G}$ и 6-31G. Расчеты выполнены с использованием программного комплекса Gaussian09 в Суперкомпьютерном центре Воронежского государственного университета.

Рассчитаны зависимости фундаментальных параметров: зазора между граничными высшей занятой (ВЗМО, или НОМО) и низшей свободной молекулярными орбиталями (HCMO, или LUMO) $\left(E_{L H}\right)$, потенциала ионизации $(I P)$, сродства к электрону $(E A)$, работы выхода электронов $(W)$, приведенной энергии связи ук-УНТ $(0,9)$ в интервале числа сегментов $i=1 . .9$, что соответствует интервалу длин 0.7-3 нм. Данные, полученные методом теории функционала плотности, демонстрируют достаточно высокое согласование с экспериментальными [10, 15, 19-24].

Положение внедрения атома примеси определено из условия минимума полной энергии при оптимизации геометрии структуры методом теории функционала плотности, полученные результаты соответствуют экспериментальным и расчетным данным других групп.

\section{РЕЗУЛЬТАТЫ РАСЧЕТОВ ЭЛЕКТРОННОЙ СТРУКТУРЫ ЧИСТЫХ НАНОТРУБКАХ}

Как было продемонстрировано ранее в работах [33], в синглетном состоянии зависимости фундаментальных параметров от длины ук-УНТ $(0,9)$ имеют монотонный характер. Потенциал ионизации монотонно уменьшается с длиной и находится в интервале $I P=5.02-7.56$ эВ и $5.12-7.56$ эВ для нанотрубок $D_{3 h} / D_{3 d}$ и $D_{3}$ симметрии. Сродство к электрону ук-УНТ $D_{3 h} / D_{3 d}$ симметрии увеличивается с ростом длины нанотрубки и соответствует интервалу $E A=2.68-4.10$ эВ. Сродство к электрону ук-УНТ $D_{3}$ симметрии, рассчитанное методом DFT, при числе сегментов $i>2$ не зависит от длины нанотрубки и находится в интервале $E A=2.68-$ 3.99 эВ.

При переходе в триплетное состояние зависимости качественно сохраняются, однако имеют ряд особенностей. Энергетические уровни молекулярных орбиталей для электронов со спином вверх и вниз в триплетном состоянии смещаются пропорционально энергии синглетного состояния, это смещение определяется также и симметрией ук-УНТ. В триплетном состоянии в трубках симметрии $D_{3}$ энергии LUMO и HOMO для электронов со спинами вверх и вниз количественно сдвигаются практически симметрично по отношению к граничным орбиталям синглетного состояния. Таким образом,

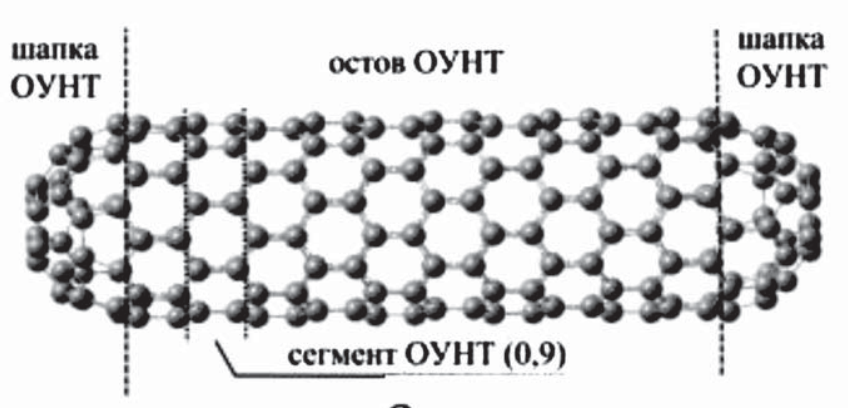

a

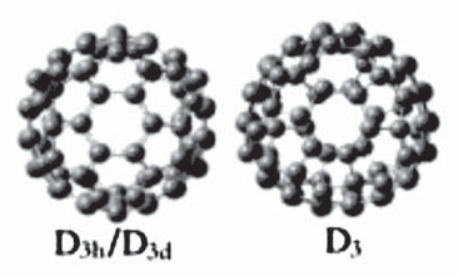

b

Рис. 1. Структура и симметрия закрытых ук-УНТ $(0,9)$, вид сбоку $(a)$ и с торца $(b)$

[Fig. 1. Structural images of capped us-CNTs $(0,9)(a)$. The mutual orientation of two $\mathrm{C}_{60}$ hemisphere caps $D_{3 h} / D_{3 d}$ and $D_{3}$ symmetries $\left.(b)\right]$ 
зависимости энергетической щели между граничными орбиталями $E_{L H}(\uparrow)$ и $E_{L H}(\downarrow)$ сходятся к близким значениям при увеличении числа сегментов.

Закономерности изменения спинзависимых транспортных свойств от длины качественно различны для трубок симметрий $D_{3 h} / D_{3 d}$ и $D_{3}$ : обнаружено, что при переходе синглет-триплет проводимость трубок симметрии $D_{3}$ снижается, что обусловлено увеличением энергетического зазора между граничными орбиталями, $E_{L H}(\uparrow)$ и $E_{L H}(\downarrow)$ изменяются на $0.1-0.5$ эВ относительно энергии $E_{L H}$ синглета.

Уменьшение зазора между граничными орбиталями ук-УНТ симметрии $D_{3 h} / D_{3 d}$ при переходе синглет-триплет приводит к несимметричному изменению энергии орбиталей. В результате энергии $\operatorname{HOMO}(\downarrow)$ и $\operatorname{LUMO}(\uparrow)$ близки к соответствующим энергиям в синглете. Уменьшение спин-зависимого зазора между граничными орбиталями составляет 0.4-1.5 эВ, что определяет существенное увеличение проводимости.

Как следует из анализа спектров свободных и занятых состояний, степень спиновой поляризации резко убывает при наращивании длины трубки, что объясняется снижением разницы между энергетическими зазорами со спинами вверх и вниз с 0.231 до 0.011 эВ при числе сегментов от 2 до 8 (симметрия $D_{3}$ ). Касаемо ук-УНТ $(0,9)$ симметрии $D_{3 h} / D_{3 d}$, максимум спиновой поляризации наблюдается при числе сегментов 4, а затем при наращивании длины происходит линейное уменьшение, разница энергетических зазоров лежит в интервале 0.056-0.107 эВ.

Полученные данные согласуются с рядом экспериментальных и теоретических работ по исследованию электронной структуры углеродных материалов [20-30].

\section{РЕЗУЛЬТАТЫ РАСЧЕТОВ ЭЛЕКТРОННОЙ СТРУКТУРЫ В ЛЕГИРОВАННЫХ (ДОПИРОВАННЫХ) НАНОТРУБКАХ}

Было установлено, что нейтральные эндоэдральные комплексы нестабильны, но демонстрируют зарядовую стабильность. В синглетном состоянии Li@ук-УНT анион также не стабилен, и литий стремится покинуть нанотрубку через фронтальный гексагон шапки. Переход в триплетное состояние стабилизирует анион. Ион лития с эффективным зарядом $\operatorname{Qeff}(\mathrm{Li})=1.014 \mathrm{e}$ ориентирован противоположно фронтальному гексагону на расстоянии $R_{\mathrm{Li-C}}=2.28 \AA$ до его атомов углерода. Атомы гексагона имеют эффективный заряд $\operatorname{Qeff}(\mathrm{C})=-0.05 e$. Спин-зависимая энергетическая щель $E_{L H}(\uparrow)=0.70-0.21$ эВ и $E_{L H}(\downarrow)=0.11-0.05$ эВ для $i=1-11$ уменьшается с увеличением длины.

При анализе результатов моделирования установлено, что $E_{L H}$ для спинов-вверх и спинов-вниз допированных трубок меньше, чем чистых, таким образом, допирующая примесь вызывает модуляцию запрещенной зоны, вследствие чего влияет на проводимость. Также стоит отметить, что для допированных комплексов $E_{L H}(\downarrow)$ слабо зависит от длины.

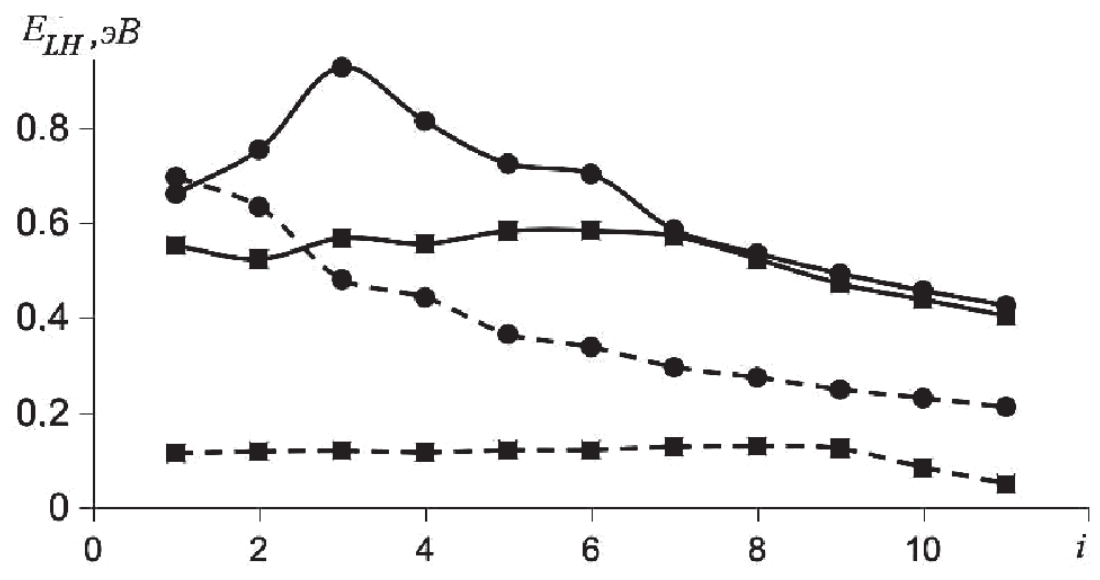

Рис. 2. Зависимость энергетического зазора между граничными орбиталями ук-УНТ, допированных анионом лития, от числе сегментов (сравнение с чистыми), круглые маркеры - электроны со спинов вверх, квадратные со спином вниз, сплошная линия - чистые ук-УНТ, пунктирная - допированные литием

[Fig. 2. The dependency of energy gap between frontier orbitals of us-CNTs doped by Li anion versus the segments number (comparing with the pure ones). Round markers correspond to the majority spin electrons, the square ones correspond to the minority spin electrons] 


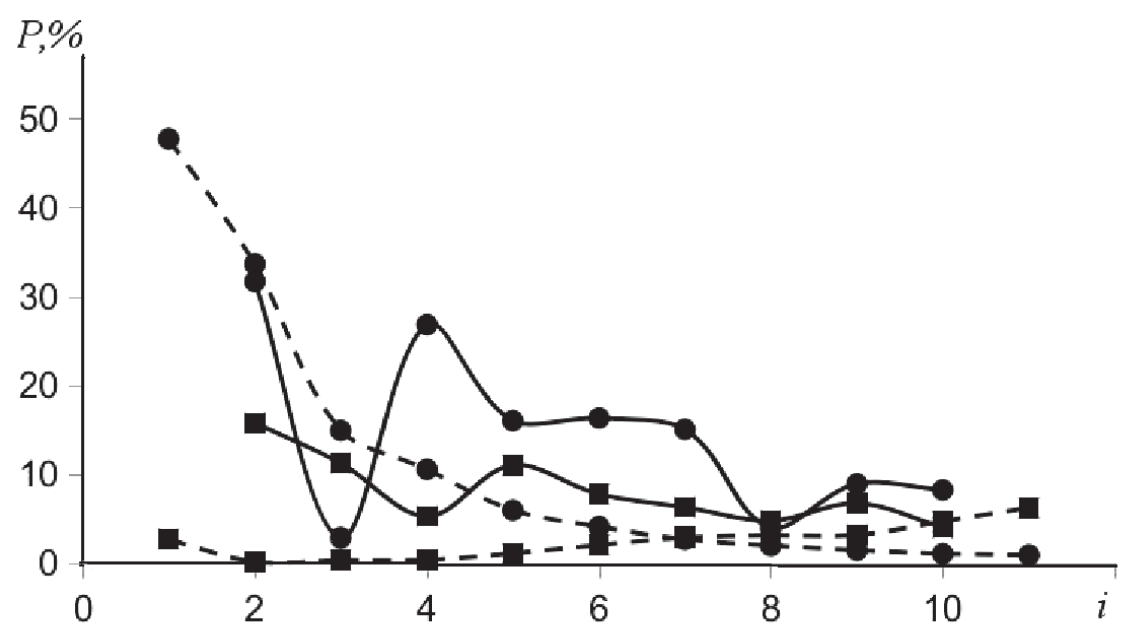

Рис. 3. Зависимость спиновой поляризации ук-УНТ, допированной анионом лития, от числа сегментов (сравнение с чистыми), круглые маркеры - электроны со спинов вверх, квадратные - со спином вниз, сплошная линия чистые ук-УНТ, пунктирная - допированные литием

[Fig. 3. The dependency of spin polarization of us-CNTs doped by Li anion versus the segments number(comparing with the pure ones). Round markers correspond to the majority spin electrons, the square ones correspond to the minority spin electrons]

Катионные и анионные комплексы термодинамически стабильны во всем исследованном интервале длин ук-УНТ. Эффективный заряд Li $Q_{e f f}(\mathrm{Li})=1.033 e$. Ион находится на расстоянии $R_{\mathrm{Li-c}}=2.28 \AA$ от отрицательно заряженных атомов гексагона, у которого атомы имеют эффективный заряд $Q_{e f f}(\mathrm{C})=-0.05 e$. Энергетический зазор уменьшается по отношению к чистым ук-УНТ $(0,9)$ и лежит в диапазоне $E_{L H}=0.78-0.23$ эВ для $i=1-11$, когда для чистых трубок 0.81-0.29 эВ, как показано ранее.

Выявлена инверсия спиновой поляризации для анионов эндоэдральных комплексов, которая определяется уменьшением спиновой поляризации с 48 до $1 \%$ для спина вверх и увеличением с 2.7 до $6.2 \%$ для спина вниз. Инверсия спиновой поляризации наблюдается для комплекса с числом сегментов $i=7$. Эффект инверсии спиновой поляризации наблюдался и описывался для чистых ук-УНТ $(0,9)$ в сильном электрическом поле [33]. Увеличение спиновой поляризации для спина вниз наблюдается только на сегментах $n=2,3$, что так же, как и в случае аниона, определяет перспективы в контролирование спиновой поляризации на маленьком количестве сегментов. Качественно зависимости сохраняются и при допировании натрием.

Наличие спин-поляризованных носителей в чистой ук-УНТ объясняется спин-орбитальным взаимодействием, ее увеличение за счет внедрен- ного атома Li можно объяснить косвенным обменным взаимодействием РККИ (взаимодействие Рудермана-Киттеля-Касуя-Иосиды) по аналогии с графеном, допированным одновалентным атомом, как описано в [32-33] в рамках $s-d$ модели, адаптированной к случаю, когда в качестве коллективизируемых выступают $p$-электроны атомов углерода, а в качестве примеси $s$-электроны водорода или щелочного металла.

\section{ЗАКЛЮЧЕНИЕ}

В рамках моделирования параметров электронной структуры изучены закономерности изменения фундаментальных параметров для ук-УНТ.

Из анализа результатов численного эксперимента следует, что в спин-триплетном состоянии как в чистых, так и в допированных трубках наблюдаются различия в величине энергетического зазора между граничными орбиталями для электронов с противоположными спинами, что говорит о спин-зависимом характере проводимости в ук-УНТ. Установлен диапазон длин, в котором данные эффекты существенны. Показано, что контролируемым введением примеси может быть осуществлена модуляция спиновой поляризации и спинзависимой проводимости. При допировании возможно наблюдение эффекта инверсии спиновой поляризации, что ранее в укУНТ описано лишь в сильных электрических и магнитных полях. 
Технологический способ модуляции спиновой поляризации позволяет контролировать спиновые свойства на этапе синтеза и расширяет сферы применения углеродных нанотрубок как функционального материала при проектировании функциональных спинтронных устройств.

Высокая поляризация вкупе со спинзависимой электропроводностью открывают перспективы создания спиновых фильтров, логических вентилей и других устройств.

Работа выполнена при финансовой поддержке РФФИ (проект № 16-32-00926 мол_а).

\section{СПИСОК ЛИТЕРАТУРЫ}

1. Bhaattacharya S., Akande A. and Sanvito S. // Chemical Communications, 2014, vol. 50, pp. 6626-6629.

2. Awschalom D. D., Flatte M. E. // Nature Communications, 2007, pp. 153-159.

3. Murat A., Rungger I., Jin C., Sanvito S. and Schwingenschlögl U. // J. Phys. Chem. C, 2014, vol. 118, pp. 3319 3323.

4. Sun L., Wei1 P., Wei J., Sanvito S. and Hou1 S. // J. Phys. Condens. Matter, 2011, vol. 23, p. 425301.

5. Wu J., and Hagelberg F. // Phys. Rev. B, 2010, vol. 81, pp. $155407-155415$.

6. Kamil L., Ritschel M., Albrecht L., Krupskaya Y., Buchner B., Klingeler R. // J. Phys.: Conf. Ser., 2010, vol. 200, pp. 072061.

7. Minot E. D., Yaish Y., Sazonova V., McEuen P. // Nature, 2004, vol. 428, p. 536.

8. Jung H. Y., Jung S. M., Kim L. // Carbon, 2008, vol. 46, pp. 969-973.

9. Sanchez-Valencia J. R., Dienel T., Gruning O., et al. // Nat. Lett., 2014, vol. 512, p. 61.

10. Kato T., Hatakeyama R. // ACS Nano, 2010, vol. 4 pp.7395-7400.

11. Tuchin A. V., Nestrugina A. V., Bityutskaya L. A., Bormontov E. N. // J. Phys.: Conf. Ser., 2014, vol. 541, p. 012008.

12. Cioslowski J., Rao N. and Moncrieff D. // J. Am. Chem. Soc., 2002, vol. 124, p. 8485.

13. Rocherfort A., Salahub D. R., et al. // J. Phys. Chem. B, 1999, vol. 103, p. 641 .

14. Buonocore F., Trani F., Ninno D., et al. // Nanotech, 2008, vol. 19, p. 025711 (6).

15. Wang B.-C., Wang H.-W., Lin I.-C., Lin Y.-S., Chou Y.-M. and Chiu H.-L. // J. Chin. Chem. Soc., 2002, vol. 50, p. 939 .
16. Tuchin A. V., Ganin A. A., Zhukalin D. A., Bitytskaya L. A. and Bormontov E. N. // Recent Advances in Biomedical \& Chemical Engineering and Materials Science, 2014, vol. 1, p. 40.

17. Lu D., Li Y., Rotkin S. V., Ravaioli U., Schulten K. // Nano Letters, 2004, vol. 4, p. 2383.

18. Yumura T., Hirahara K., Bandow S., et. al. // Chemical Physics Letters, 2004, vol. 386, p. 38.

19. Parker S. F., Bennington S. M., Taylor J. W., Herman H., Silverwood I., Albers P., Refson K. // Phys. Chem. Chem. Phys., 2011, vol. 13, p. 11192.

20. Tuchin A. V., Bityutskaya L. A., Bormontov E. N. // Eur. Phys. J. D, 2015, pp. 69-87.

21. Schettino V., Pagliai M., and Cardini G. // J. Phys. Chem.: A, 2002, vol. 106, p. 1815.

22. Hertel I. V., Steger H., de Vries J., Weisser B., Menzel C., Kamke W. // Phys. Rev. Lett., 1992, vol. 68, p. 784.

23. Yoo R. K., Ruscic B., Berkowitz J. // J. Chem. Phys., 1992, vol. 96, p. 911.

24. de Vries J., Steger H., Kamke B., Menzel C., Weisser B., Kamke W., Hertel I. V. // Chemical Physics Letters, 1992, vol. 188, p. 159.

25. Steger H., Holzapfel J., Hielscher A., Kamke W., Hertel I. V. // Chemical Physics Letters, 1995, vol. 234, p. 455.

26. Brink C., Andersen L. H., Hvelplund P., Mathur D., Voldstad J. D. // Chemical Physics Letters, 1995, vol. 233, p. 52.

27. Wang X. B., Ding C. F. and Wang L. S. // J. Chem. Phys., 1999, vol. 110, p. 8217.

28. Dresselhaus M. S., Dresselhaus G. and Saito R. // Carbon, 1995, vol. 33, p. 883.

29. Saito R., Fujita M., Dresselhaus G. and Dresselhaus

M. // Phys. Rev. B, 1992, vol. 46, p. 1804.

30. Odom T. W., Huang J. L., Kim P. and Lieber C. M. // Nature, 1998, vol. 391, p. 62.

31. Ouyang M., Huang J. L. and Lieber C. M. // Acc. Chem. Res, 2002, vol. 35 p. 1018.

32. Белоненко М. Б., Лебедев Н. Г., Пак А. В. // ФTT, 2011, т. 53, № 8, c. 1604-1608.

33. Белоненко М. Б., Лебедев Н. Г., Пак А. В. // Письма в ЖТФ, 2011, т. 37, № 15, с. 69-78.

34. Глушков Г. И., Тучин А. В., Ефимов Н. Н., Бормонтов Е. Н. // Конденсированные среды имежсазные гранищы, 2016, т. 19, № 1, с. 37-41.

35. Glushkov G. I., Tuchin A. V., Bityutskaya L. A. // Journal of Nano and Microsystem Technique, 2016, vol. 18, pp. 531- 538,

36. Durgun E., Dag S., Bagci V. M. K., Gulseren O. // Phys. Rev. B, 2003, vol. 67, pp. 201401-201404. 


\title{
MODULATION OF THE ELECTRINIC STRUCTURE OF ULTRA-SHORT CARBON NANOTUBES $(0,9)$ DOPED BY ALKALIC METALS
}

\author{
(C) 2017 G. I. Glushkov, A. V. Tuchin, E. N. Bormontov \\ Voronezh State University, 1 Universitetskaya sq., 394018, Voronezh, Russia \\ e-mail: green5708@yandex.ru \\ Received 14.08.2017
}

\begin{abstract}
The main task for modern spintronics is to find materials with high spin polarization that enable spin transport. Carbon nanomaterials are under special focus, including ultra-short carbon nanotubes (us-CNTs) which are thermodynamically stable and have high spin polarization. Synthesis methods for us-CNTs with a narrow distribution of the tube length and chirality have recently been developed.

The paper describes a numerical simulation experiment that was carried out by means of the method based on density functional theory. Its goal was to calculate the main electron structure parameters of alkali-metal doped us-CNTs to determine the conditions of intrinsic spin polarization.

It was established that in a close interval the $3 \mathrm{k}$-rule does not work for metal tubes $(0,9)$. There is a non-zero gap between the frontier molecular orbitals and the tube acts as a semiconductor. The gap between the highest occupied and the lowest unoccupied molecular orbitals depends on electron spin at the triplet state, thus the conductivity is spin-dependent, which makes it possible to use us-CNTs to construct transport devices. The energy gap decreases with an increase in tube length.

The controlled alkali metal doping modulates the energy gap that leads to the increase in conductivity and spin polarization in case of anion complexes.

Thus, the paper describes a technological way to modify the properties of us-CNTs to create functional spintronic devices.
\end{abstract}

Keywords: spin, spintronics, carbon nanotube, doping, alkali metals.

\section{ACKNOWLEDGEMENTS}

The reported study was supported by the Russian Foundation for Basic Research (project No.6-3200926 mol_a).

\section{REFERENCES}

1. Bhaattacharya S., Akande A. and Sanvito S. Chemical Communications, 2014, vol. 50, pp. 6626-6629. DOI: 10.1039/C4CC01710B

2. Awschalom D. D., Flatte M. E. Nature Communications, 2007, pp. 153-159. DOI: 10.1038/nphys551

3. Murat A., Rungger I., Jin C., Sanvito S. and Schwingenschlögl U. J. Phys. Chem. C, 2014, vol. 118, pp. 33193323. DOI: $10.1021 / \mathrm{jp} 4100153$

4. Sun L., Wei1 P., Wei J., Sanvito S. and Hou1 S. J. Phys. Condens. Matter, 2011, vol. 23, p. 425301.

5. Wu J., and Hagelberg F. Phys. Rev. B, 2010, vol. 81, pp. 155407-155415.

6. Kamil L., Ritschel M., Albrecht L., Krupskaya Y., Buchner B., Klingeler R. J. Phys.: Conf. Ser., 2010, vol. 200, pp. 072061. DOI:10.1088/1742-6596/200/7/072061

7. Minot E. D., Yaish Y., Sazonova V., McEuen P. Nature, 2004, vol. 428, p. 536.

8. Jung H. Y., Jung S. M., Kim L. Carbon, 2008, vol. 46, no. 6, pp. 969-973. https://doi.org/10.1016/j.carbon.2008.03.006
9. Sanchez-Valencia J. R., Dienel T., Gruning O., et al. Nat. Lett., 2014, vol. 512, p. 61. DOI: 10.1038/nature13607.

10. Kato T., Hatakeyama R. ACS Nano, 2010, vol. 4 pp.7395-7400. DOI: $10.1021 / \mathrm{nn} 102379$ p

11. Tuchin A. V., Nestrugina A. V., Bityutskaya L. A., Bormontov E. N. J. Phys.: Conf. Ser., 2014, vol. 541, p. 012008. DOI: $10.1088 / 1742-6596 / 541 / 1 / 012008$

12. Cioslowski J., Rao N. and Moncrieff D. J. Am. Chem. Soc., 2002, vol. 124, p. 8485. DOI: $10.1021 /$ ja0126879

13. Rocherfort A., Salahub D. R., et al. J. Phys. Chem. $B, 1999$, vol. 103, p. 641. DOI: 10.1021/jp983725m

14. Buonocore F., Trani F., Ninno D., et al. Nanotech, 2008, vol. 19, p. 025711 (6). DOI: 10.1088/09574484/19/02/025711.

15. Wang B.-C., Wang H.-W., Lin I.-C., Lin Y.-S., Chou Y.-M. and Chiu H.-L. J. Chin. Chem. Soc., 2002, vol. 50, p. 939.

16. Tuchin A. V., Ganin A. A., Zhukalin D. A., Bitytskaya L. A. and Bormontov E. N. Recent Advances in Biomedical \& Chemical Engineering and Materials Science, 2014, vol. 1, p. 40.

17. Lu D., Li Y., Rotkin S. V., Ravaioli U., Schulten K. Nano Letters, 2004, vol. 4, p. 2383. 
18. Yumura T., Hirahara K., Bandow S., et. al. Chemical Physics Letters, 2004, vol. 386, p. 38. https://doi. org/10.1016/j.cplett.2003.12.123

19. Parker S. F., Bennington S. M., Taylor J. W., Herman H., Silverwood I., Albers P., Refson K. Phys. Chem. Chem. Phys., 2011, vol. 13, pp. 7789-7804. http://dx.doi. org/10.1039/C0CP02956D

20. Tuchin A. V., Bityutskaya L. A., Bormontov E. N. Eur. Phys. J. D, 2015, pp. 69:87. DOI: 10.1140/epjd/e201550440-2

21. Schettino V., Pagliai M., and Cardini G. J. Phys. Chem.: A, 2002, vol. 106, p. 1815. DOI: 10.1021/ jp012680d

22. Hertel I. V., Steger H., de Vries J., Weisser B., Menzel C., Kamke W. Phys. Rev. Lett., 1992, vol. 68, p. 784. DOI:https://doi.org/10.1103/PhysRevLett.68.784

23. Yoo R. K., Ruscic B., Berkowitz J. J. Chem. Phys., 1992, vol. 96, p. 911.

24. de Vries J., Steger H., Kamke B., Menzel C., Weisser B., Kamke W., Hertel I. V. Chemical Physics Letters, 1992, vol. 188, p. 159. https://doi.org/10.1016/00092614(92)90001-4

25. Steger H., Holzapfel J., Hielscher A., Kamke W., Hertel I. V. Chemical Physics Letters, 1995, vol. 234, p. 455. https://doi.org/10.1016/0009-2614(94)01476-C

26. Brink C., Andersen L. H., Hvelplund P., Mathur D., Voldstad J. D. Chemical Physics Letters, 1995, vol. 233, p. 52. https://doi.org/10.1016/0009-2614(94)01413-P
27. Wang X. B., Ding C. F. and Wang L. S. J. Chem. Phys., 1999, vol. 110, p. 8217. https://doi.org/10.1063/ 1.478732

28. Dresselhaus M. S., Dresselhaus G. and Saito R. Carbon, 1995, vol. 33, p. 883. https://doi.org/10.1016/00086223(95)00017-8

29. Saito R., Fujita M., Dresselhaus G. and Dresselhaus M. Phys. Rev. B, 1992, vol. 46, p. 1804. DOI:https://doi. org/10.1103/PhysRevB.46.1804

30. Odom T. W., Huang J. L., Kim P. and Lieber C. M. Nature, 1998, vol. 391, p. 62. doi:10.1038/34145

31. Ouyang M., Huang J. L. and Lieber C. M. Acc. Chem. Res, 2002, vol. 35 p. 1018.

32. Belonenko M. B., Lebedev N. G., Pak A. V. Physics of the Solid State, 2011, vol. 53, № 8, pp. 1604-1608. Available at: http://journals.ioffe.ru/articles/viewPDF/1530 (in Russian)

33. Belonenko M. B., Lebedev N. G., Pak A. V. Technical Physics Letters, 2011, vol. 37, no. 8, pp. 724-727. DOI: 10.1134/S1063785011080049

34. Glushkov G. I., Tuchin A. V., Efimov N. N., Bormontov E. N. Condensed Matter and Interphases, 2016, vol. 19, no. 1, pp. 37-41. Available at: http://www.kcmf. vsu.ru/resources/t_19_1_2017_004.pdf

35. Glushkov G. I., Tuchin A. V., Bityutskaya L. A. Journal of Nano and Microsystem Technique, 2016, vol. 18, pp. 531- 538 .

36. Durgun E., Dag S., Bagci V. M. K., Gulseren O. Phys. Rev. B, 2003, vol. 67, pp. 201401-201404.
Глушков Григорий Иванович - аспирант кафедры физики полупроводников и микроэлектроники, Воронежский государственный университет; тел.: +7 (952) 5461253, e-mail: green5708@yandex.ru

Тучин Андрей Витальевич - к. ф.-м. н, доцент кафедры физики полупроводников и микроэлектроники, Воронежский государственный университет; тел.: +7 (908) 1485775, e-mail: a.tuchin@bk.ru

Бормонтов Евгений Николаевич - д. ф.-м. н., профессор, заведующий кафедрой физики полупроводников и микроэлектроники, Воронежский государственный университет; тел.: +7 (473) 2208481, e-mail: me144@ phys.vsu.ru
Glushkov Grigory I. - postgraduate student, Department of Physics of Semiconductors and Microelectronics, Voronezh State University; ph.: +7 (952) 5461253, e-mail: green5708@yandex.ru

Tuchin Andrei V. - Cand. Sci. (Phys.-Math.), Assistant Professor, Department of Physics of Semiconductors and Microelectronics, Voronezh State University; ph.: +7 (908) 1485775, e-mail: a.tuchin@bk.ru

Bormontov Eugene N. - Dr. Sci. (Phys.-Math.), Head of Department of Physics of Semiconductors and Microelectronics, Voronezh State University; ph.: +7 (473) 2208481, e-mail: me144@phys.vsu.ru 